hep-th/0207226

SU-ITP-02/31

\title{
Lattice Gauge Theories
}

\author{
have \\ Gravitational Duals
}

\author{
Simeon Hellerman ${ }^{1,2}$ \\ ${ }^{1}$ Department of Physics, Stanford University, Stanford, CA 94305 \\ ${ }^{2}$ SLAC Theory Group, MS 81, PO Box 4349, Stanford, CA 94309
}

\begin{abstract}
In this paper we examine a certain threebrane solution of type IIB string theory whose long-wavelength dynamics are those of a supersymmetric gauge theory in $2+1$ continuous and 1 discrete dimension, all of infinite extent. Low-energy processes in this background are described by dimensional deconstruction, a strict limit in which gravity decouples but the lattice spacing stays finite. Relating this limit to the near-horizon limit of our solution we obtain an exact, continuum gravitational dual of a lattice gauge theory with nonzero lattice spacing. $H$-flux in this translationally invariant background encodes the spatial discreteness of the gauge theory, and we relate the cutoff on allowed momenta to a giant graviton effect in the bulk.
\end{abstract}

July 24, 2002 


\section{Introduction}

The modern approach to string theory rests on two conceptual foundations.

The first is that string theories in Minkowski space can be derived as limits of conventional quantum theories as the number of degrees of freedom per unit volume becomes large. The second foundation is that one need not take a strict limit in order to obtain a theory with a gravitational interpretation. Many different gauge theories whose large-n limits yield string or M theory in ten or eleven flat dimensions have finite-n versions which correspond to backgrounds with mutually distinct geometries. The size scales of these geometries grow as positive powers of the number of degrees of freedom per site - which typically means that the size grows as a positive power of the rank $n$ of a gauge group.

These two foundations, now established beyond dispute, lead one to wonder whether all quantum theories with large numbers of degrees of freedom might have some kind of string-theoretic interpretation and, if not, what the criterion could be for a nongravitational theory to have a gravitational dual. These important questions will not be answered here. Rather, we propose to enlarge dramatically the class of quantum theories which admit gravitational duals, perhaps enlarging that class so much as to plant in the reader's mind some doubt that there may be any Hamiltonian it does not contain.

Most gravity/gauge theory dualities proposed so far have had two common and related features. On the nongravitational side we have local quantum field theories, forumulated in the continuum without a cutoff 1 On the gravitational side, asymptotically $A d S$ geometries encode the fact that the nongravitational description has conformal symmetry in the ultraviolet, which some would consider the definitive criterion of a quantum field theory. Asymptotically $A d S_{D+1}$ boundary conditions also express the condition that the entropy is extensive on the $D$-dimensional boundary but not in the bulk, as illustrated in [5], in accordance with the holographic principle.

The second foundation of string theory expressed as above leads inevitably to a certain question. There is a point of view from which gauge theories in the continuum, even at finite $n$, should themselves be considered as limits. Namely they like all QFT's are limits of theories regulated with an ultraviolet cutoff at an energy scale $\Lambda$, which is later taken to

1 Exceptions to this pattern are proposed dualities for noncommutative quantum field theories, tensionless string theories, and 'little string theories' ([1], [2], [3], [4]). In this discussion we would like to focus on quantum theories which one knows how to regulate and define independently of string theory. 
$\infty$. If the cutoff is a consistent, unitary quantum regulator such as a Hamiltonian lattice theory with spacing $\Lambda^{-1}$, one is led to ask: does the regulated theory with finite cutoff admit its own consistent gravitational interpretation?

We answer in the affirmative, making the following points:

- Lattice gauge theories with finite spacing have exact, continuum gravitational duals.

- Worldsheet instantons encode the gauge theory's spatial discreteness, in the form of momentum-nonconserving, or umklapp, processes.

- The maximal size of a giant graviton in the bulk imposes an upper bound on the momenta of composite particles in the gauge theory.

The specific case we consider is the case of $D=4, N=4$ super-Yang-Mills theory with one of the three spatial dimensions discretized, which breaks the supersymmetry down by half. We obtain the gauge theory, and the associated supergravity solution, by starting with a D-brane configuration in type II string theory whose long-wavelength dynamics are described by this discretized gauge theory, and then taking a limit in which gravity decouples but the lattice spacing stays finite. We note also that at infinite 't Hooft coupling, our work provides a precise realization of the ideas of [6]. At finite 't Hooft coupling, the picture of [6] is corrected due to the presence of the flux and negative spatial curvature in the bulk.

After reviewing some background in section two, we perform the decoupling limit in section three; in section four we identify the stringy processes which encode momentum nonconservation; in section five we find that the upper bound on the momentum of composit states is enforced by a 'giant graviton' effect in the bulk; and in section six we discuss possible applications and directions for further study.

\section{Dimensional deconstruction is $T$-duality}

We begin with $n$ D2-branes near the fixed point of a $\mathbb{Z}_{k}$ orbifold of $\mathbb{C}^{2}$. First we review, along the lines of [7], [8] the low-energy dynamics of the brane sector of this theory and show that they are those of a gauge theory on a finite periodic lattice.

We discuss scales and couplings in the theory, and two independent limits one can take, the first of which corresponds to infinite volume in the lattice gauge theory, and the second of which corresponds to the limit in which gravity decouples from the gauge theory. In this section we will discuss only the first of the two. We will also use $T$-duality to construct an equivalent background of type IIB string theory. 


\subsection{D2-branes probing orbifolds}

We now study the theory of $n$ D2-branes near, but not coincident with, an $A_{k}$ orbifold singularity in string theory. First we consider branes probing the covering space.

The low-energy behavior of a set of coincident twobranes in flat space at weak string coupling is described by a $2+1$-dimensional gauge theory with sixteen supercharges, which has a unique renormalizable action. Decomposing the matter into multiplets under $N=4$ SUSY in 3 dimensions, we have a gauge multiplet and a single hyperultiplet in the adjoint representation of the gauge group $U(n k)$. The vector multiplet contains a gauge field $\hat{A}_{\mu}$, three adjoint scalars $\hat{Z}^{A}$ and four Majorana fermions $\hat{\lambda}_{\alpha}$. The hypermultiplet contains four scalars $\hat{Y}^{i}$ in the adjoint, and four Majorana fermions $\hat{\psi}_{\alpha}$, also in the adjoint.

The Lagrangian is

$$
\begin{aligned}
& g_{Y M 3}^{2} \mathcal{L}=-\operatorname{tr} \hat{F}_{\mu \nu} \hat{F}^{\mu \nu}-\frac{1}{2}\left(\nabla_{\mu} \hat{Z}^{A}\right)\left(\nabla^{\mu} \hat{Z}^{A}\right)-\frac{1}{2}\left(\nabla_{\mu} \hat{Y}^{i}\right)\left(\nabla_{\mu} \hat{Y}^{i}\right) \\
&+\frac{1}{4} \operatorname{tr}\left[\hat{Z}^{A}, \hat{Z}^{B}\right]^{2}+ \frac{1}{4} \operatorname{tr}\left[\hat{Y}^{i}, \hat{Y}^{j}\right]^{2}+\frac{1}{2} \operatorname{tr}\left[\hat{Y}^{i}, \hat{Z}^{A}\right]^{2} \\
&+ \text { fermions }
\end{aligned}
$$

The orbifolded theory is obtained ([9], [10]) by truncating the fields of this Lagrangian to the set invariant under the combined action of a global rotation on the hypermultiplets:

$$
\left[\begin{array}{c}
\hat{Y}_{1}+i \hat{Y}_{2} \\
\hat{Y}_{3}+i \hat{Y}_{4}
\end{array}\right] \rightarrow\left[\begin{array}{cc}
\exp \left\{\frac{2 \pi i}{k}\right\} & 0 \\
0 & \exp \left\{-\frac{2 \pi i}{k}\right\}
\end{array}\right]\left[\begin{array}{l}
\hat{Y}_{1}+i \hat{Y}_{2} \\
\hat{Y}_{3}+i \hat{Y}_{4}
\end{array}\right]
$$

and a gauge transformation acting on the first tensor factor of the gauge indices:

$$
\hat{Y}_{p p^{\prime} \mid q q^{\prime}} \rightarrow \exp \left\{\frac{2 \pi i(p-q)}{k}\right\} \hat{Y}_{p p^{\prime} \mid q q^{\prime}}
$$

The components of the vector multiplets that survive the truncation are the blocks on the diagonal:

$$
\begin{gathered}
\hat{Z}_{p p^{\prime} \mid q q^{\prime}}^{A}=\left\{\begin{array}{cc}
0, & p \neq q \\
z_{p^{\prime} q^{\prime}}^{A}(p), & p=q
\end{array}\right\} \\
\hat{A}_{\mu, p p^{\prime} \mid q q^{\prime}}=\left\{\begin{array}{cc}
0, & p \neq q \\
A_{\mu, p^{\prime} q^{\prime}}(p), & p=q
\end{array}\right\}
\end{gathered}
$$

That is, they are adjoints of individual $U(n)$ factors of $U(n)^{k}$. The surviving hypers are bifundamentals under adjacent $U(n)$ 's; that is,

$$
\hat{Y}^{6}+i \hat{Y}_{p p^{\prime} \mid q q^{\prime}}^{7}=\left\{\begin{array}{cc}
0, & p \neq q+1 \\
\left(y^{6}+i y^{7}\right)_{p^{\prime} q^{\prime}}(p), & p=q+1
\end{array}\right\}
$$




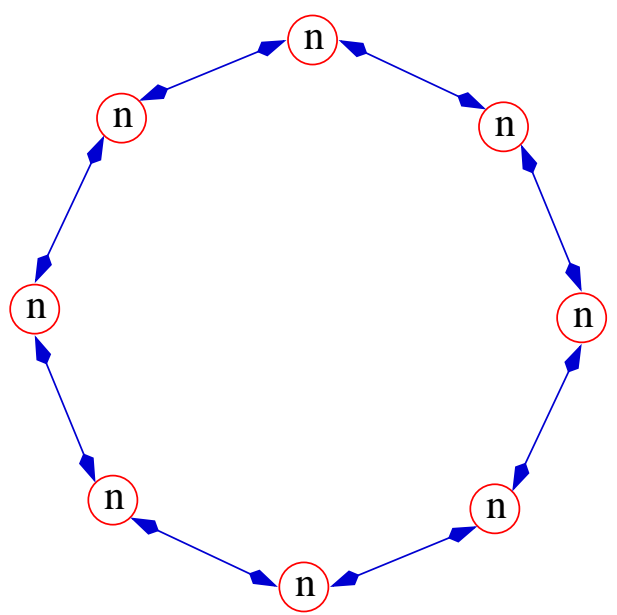

Fig. 1: Quiver for branes probing a $\mathbb{Z}_{k}$ orbifold of $\mathbb{C}^{2}$ (here $k=8$ ). The circles repreresent $U(n)$ gauge groups and the double-ended arrows represent hypermultiplets in the $(n, \bar{n})$ of adjacent gauge groups. There is also an eight-supercharge vector multiplet for each gauge group, which we have not shown explicitly.

and similarly for $\hat{Y}^{8}-i \hat{Y}^{9}$. The surviving matter content is summed up in the quiver diagram in figure 1.

If we Higgs the gauge group down to $U(n)$ by giving the hypers a vev

$$
\begin{aligned}
& \left(y^{6}+i y^{7}\right)_{p^{\prime} q^{\prime}}(p)=v_{1} \delta_{p^{\prime} q^{\prime}} \\
& \left(y^{8}+i y^{9}\right)_{p^{\prime} q^{\prime}}(p)=v_{2} \delta_{p^{\prime} q^{\prime}},
\end{aligned}
$$

then the spectrum of massive fluctuations about the vacuum is

$$
E_{j}=v \sin (\pi j / k), \quad j=0, \cdots, k-1
$$

with $v \equiv\left(\left|v_{1}\right|^{2}+\left|v_{2}\right|^{2}\right)^{\frac{1}{2}}$. These fluctuations correspond to the lightest twisted open strings on the D2-branes, T-dual to discrete momentum eigenmodes on the discretized type IIB D3-brane.

The construction of the discretized threebrane from a D2-brane probe of an orbifold proceeds in exact parallel to the discussion of [8]. Our theory is just a dimensional reduction of theirs at weak coupling, along one of the ordinary continuous dimensions.

\subsection{Scales and couplings of the probe theory}

Ultimately we will wish to take two logically independent limits, corresponding to two hierarchical separations of mass scales: the first, between the string scale and the scale of 
the heaviest discrete momentum modes, and the second, between the scale of the heaviest discrete momentum modes and the scale of the lightest eigenmodes of discrete translation, or "Bloch waves".

The Bloch waves on the type IIB threebrane are T-dual, under a mod- $k$ version of the standard momentum/winding duality, to the twisted open strings of the type IIA twobrane, and their dispersion relation can be derived from looking at the lengths of the type IIA twisted strings and multiplying by the string tension $T_{\text {string }} \equiv\left(2 \pi \alpha^{\prime}\right)^{-1}$. The distance between the $j \underline{\text { th }}$ and $j^{\prime} \underline{\text { th }}$ brane clump images is just the chordal distance $2 r_{0}\left|\sin \left(\frac{\pi\left(j-j^{\prime}\right)}{k}\right)\right|$ and so the rest energy of the corresponding twisted string is $E=\frac{r_{0}}{\pi \alpha^{\prime}}\left|\sin \left(\frac{\pi\left(j-j^{\prime}\right)}{k}\right)\right|$. The dispersion relation for Bloch waves in a free field theory with nearest neighbor kinetic terms is

$$
E=\frac{\Lambda}{\pi} \sin \left(\frac{\pi\left|j-j^{\prime}\right|}{k}\right)
$$

for some mass scale $\Lambda$ which we determine in terms of the lattice spacing as follows. The lowest nonzero mode has energy $E_{\text {lowest }} \sim \Lambda / k$. At long distances, the discretization is invisible, and the lowest Bloch wave looks like a continuum fourier mode of the form $\exp \left\{2 \pi i \tilde{x}_{3} / V\right\}$, where $V \equiv k a$ is the size of the discrete direction $\tilde{x}_{3}$ and $a$ is the lattice spacing. Then $E_{\text {lowest }}=P_{\text {lowest }} \sim 2 \pi / V=2 \pi /(k a)$ where $a$ is the lattice spacing and $V \equiv k a$ is the total size of the discrete direction. So $\Lambda=k / V=1 / a$. The energy $E_{\text {highest }}$ of the highest Bloch wave is $\Lambda / \pi$.

Now we express these quantities in terms of string theory. The separation between adjacent branes is approximately given by their angular separation (on the covering space) times their separation $r_{0}$ from the origin. Their angular separation is $2 \pi / k$ and so the energies of the lightest Bloch waves go as $E_{\text {lowest }}=2 \pi r_{0} T_{\text {string }} / k=r_{0} /\left(k \alpha^{\prime}\right)=\Lambda / k$. The energies of the heaviest Bloch waves go as $2 r_{0} T_{\text {string }}=r_{0} /\left(\pi \alpha^{\prime}\right)=\Lambda / \pi$.

To summarize:

$$
\begin{gathered}
\Lambda_{\text {string }}=\frac{1}{\sqrt{\alpha^{\prime}}} \\
\Lambda \equiv \pi E_{\text {highest }}=\frac{r_{0}}{\alpha^{\prime}}=\frac{2 \pi}{a}=\frac{2 \pi k}{V} \\
\Lambda_{\mathrm{IR}} \equiv E_{\text {lowest }}=\frac{r_{0}}{k \alpha^{\prime}}=\frac{2 \pi}{k a}=\frac{2 \pi}{V}=\frac{\Lambda}{k}
\end{gathered}
$$

The first hierarchy is therefore

$$
\Lambda_{\text {string }} / \Lambda=\sqrt{\alpha^{\prime}} / r_{0}
$$


and taking it to be large is the usual decoupling limit. The second of the two hierarchies is

$$
\Lambda / \Lambda_{\mathrm{IR}}=k
$$

and taking this ratio to be large corresponds to the large-volume limit of a discretized $3+1$ dimensional gauge theory in which the dimension which is discrete is also finite finite in extent.

These two limits do not commute. Were we to take the decoupling limit first, we would simply obtain the sixteen supercharge gauge theory in $2+1$ dimensions whose supergravity dual is $M$ theory on $A d S_{4} \times S^{7}$ with $n$ units of four-form flux on the $A d S_{4}$.

Instead, we will first take $k$ large with the energies of the heaviest Bloch waves held fixed. This will yield a $3+1$ dimensional gauge theory coupled to gravity with one discretized dimension. Only afterwards, once we have understood this undecoupled lattice theory with a strictly infinite number of lattice points will we take the decoupling limit.

\subsection{Running of the gauge coupling in the undecoupled gauge theory}

Between the string scale and the scale of the discrete momentum modes, the nearestneighbor kinetic terms in the discretized direction can be ignored, and the classical scaling of the gauge coupling is that of a $3 D$ gauge theory. The effective coupling of a $3 D$ gauge theory at weak coupling runs as $\Lambda_{1} g_{Y M 3}^{2}{ }^{\left[\Lambda_{1}\right]}=\Lambda_{2} g_{Y M 3}^{2}{ }^{\left[\Lambda_{2}\right]}$ plus higher perturbative corrections. In this case we expect

$$
\Lambda g_{Y M 3}^{2}{ }^{[\Lambda]}=\Lambda_{\text {string }} g_{Y M 3}^{2}{ }^{\left[\Lambda_{\text {string }}\right]} .
$$

The other thing to be done is to translate the $4 \mathrm{D}$ coupling at the lattice scale into the 3D coupling. The translation is simple and can be seen by discretizing the lagrangian explicitly and restoring a canonical normalization for the 3D fields. This can be done in a simple scalar model with a $g^{2} \phi^{4}$ coupling - the scaling is the same. The relationship is simply $g_{3}^{2[\Lambda]}=\Lambda g_{4}^{2}$ at the scale $\Lambda$. So:

$$
g_{Y M 4}^{2}=\Lambda g_{Y M 3}^{2}{ }^{[\Lambda]}=\Lambda_{\text {string }} g_{Y M 3}^{2}{ }^{\left[\Lambda_{\text {string }}\right]}
$$

We will see later that this matches the running of the coupling in the string theory before backreaction is taken into account; the spatial dependence of the type IIB dilaton is unaffected by the backreaction of the branes. (This is special to the case of the discretized threebrane and does not hold for other dimensionally deconstructed branes.)

\subsection{T-duality of the large- $k$ orbifold}

We are first assuming we are in the 'probe' regime, in which the backreaction of the branes on the geometry is neglected. 


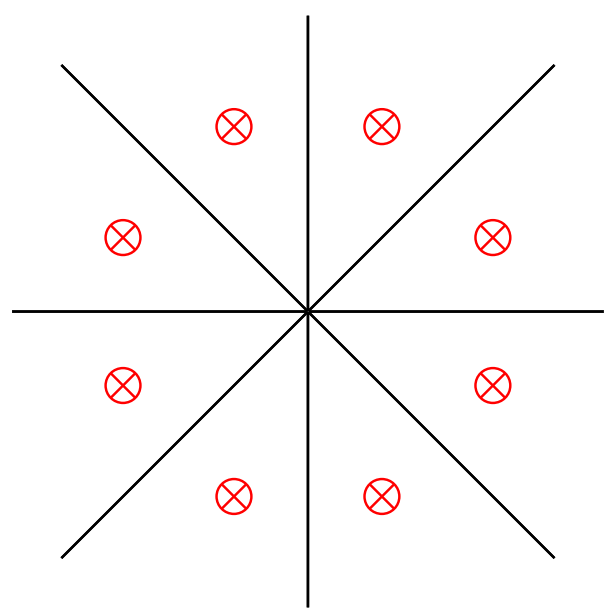

Fig. 2: Large- $k$ orbifold of $\mathbb{C}^{2}$. In the diagram the branes are represented by circles with $\times$ 's in them, $\mathbb{C}^{2}$ is represented by the plane and $k$ is represented by the number 8 .

The metric on the orbifold is given by

$$
\begin{gathered}
d s^{2}=\eta_{\mu \nu} d x^{\mu} d x^{\nu}+d z^{A} d z^{A} \\
+\left[d r^{2}+\frac{r^{2}}{k^{2}}\left(d \beta+A_{\phi}^{[\beta]} d \phi\right)^{2}+\frac{1}{4} r^{2} d \Omega_{2}^{2}\right]
\end{gathered}
$$

Now we $T$-dual along the $\beta$ direction [11]. If our new, $T$-dual angular coordinate $\gamma$ also has periodicity $\gamma \sim \gamma+2 \pi$, then our new metric is:

$$
d \tilde{s}^{2}=\eta_{\mu \nu} d x^{\mu} d x^{\nu}+\frac{k^{2} \alpha^{2}}{r^{2}} d \gamma^{2}+d z^{A} d z^{A}+d r^{2}+\frac{1}{4} d \Omega_{2}^{2}
$$

and the transformed dilaton and NS-NS two-form are given by

$$
\begin{gathered}
\exp \{2 \tilde{\Phi}\}=\frac{k^{2} \alpha^{\prime} g_{s}^{2}}{r^{2}}=\frac{\alpha^{\prime} \tilde{g}_{s}^{2}}{r^{2}} \\
\tilde{B}_{M N} d x^{M} \wedge d x^{N}=k \alpha^{\prime}(1-\cos \theta) d \phi \wedge d \gamma
\end{gathered}
$$

where $\tilde{g}_{s} \equiv k g_{s}$.

Finally we make a change of variables

$$
\tilde{x}_{3}=k \gamma / \Lambda=\gamma / \Lambda_{\mathrm{IR}}=k \gamma \alpha^{\prime} / r_{0}
$$

so that the coordinate length $V \equiv 2 \pi / \Lambda_{\text {IR }}$ of $\tilde{x}_{3}$ goes to infinity with $k$. We have chosen the normalization of $\tilde{x}_{3}$ in such a way that the enhanced Lorentz invariance in the infrared acts 
on the slice of the geometry at the location $r=r_{0}$ of the branes by rotating $\left(x^{0}, x^{1}, x^{2}, \tilde{x}_{3}\right)$ as a four-vector of $S O(3,1)$ in the usual way.

Note that with this choice, for finite $k$ the periodicity of $\tilde{x}_{3}$ is

$$
\tilde{x}_{3} \sim \tilde{x}_{3}+V
$$

so the newly defined coordinate $\tilde{x}_{3}$ can indeed be identified with the discrete compact direction in the gauge theory.

In these coordinates the type IIB string frame metric, NS-NS $B$-field, and dilaton are given by

$$
\begin{gathered}
d \tilde{s}^{2}=\eta_{\mu \nu} d x^{\mu} d x^{\nu}+\frac{r_{0}^{2}}{r^{2}} d \tilde{x}_{3}^{2}+d z^{A} d z^{A}+d r^{2}+\frac{1}{4} r^{2} d \Omega_{2}^{2} \\
\tilde{B}_{M N} d X^{M} \wedge d X^{N}=\alpha^{\prime} \Lambda(1-\cos ) \theta \cdot d \phi \wedge d \tilde{x}_{3} \\
\exp \{2 \tilde{\Phi}\}=\frac{\alpha^{\prime} \tilde{g}_{s}^{2}}{r^{2}}
\end{gathered}
$$

In terms of tilde'd quantities, the $k \rightarrow \infty$ limit is smooth.

Let us take a look at what is happening to the gauge coupling after we T-dual. 2 The dilaton in the type IIA solution varies as $\exp \{\Phi\}=g_{s}=$ const. The action of T-duality on the dilaton is

$$
\exp \{\tilde{\Phi}\}=\exp \{\Phi\} \cdot \frac{\alpha^{\prime \frac{1}{2}}}{R_{\gamma}}=g_{s} \cdot \frac{\alpha^{\prime \frac{1}{2}} k}{r}=\tilde{g}_{s} \cdot \frac{\alpha^{\prime \frac{1}{2}}}{r}
$$

The radial position of the threebranes is $r=r_{0}=\frac{\alpha^{\prime}}{a}=\alpha^{\prime} \Lambda$. So the $4 \mathrm{D}$ gauge coupling is given by

$$
g_{Y M 4}^{2}=\exp \{\tilde{\Phi}\}_{\left.\right|_{r=r_{0}}}=g_{s} \cdot \frac{\alpha^{\prime \frac{1}{2}} k}{v}=\frac{k g_{s}^{[\mathrm{IIA}]} a}{\alpha^{\prime \frac{1}{2}}}=\frac{k g_{s}}{\alpha^{\prime \frac{1}{2}} \Lambda}
$$

We now wish to take $k$ large while varying $g_{s}$ in such a way that $g_{Y M 4}^{2}$ stays fixed. While this is a rather artificial exercise from the point of view of string theory, this limit is entirely natural from the point of view of lattice gauge theory, as we explained earlier. To do this we eliminate $g_{s}$ in terms of $g_{Y M 4}^{2}$ :

$$
\begin{gathered}
g_{s} \equiv \frac{\alpha^{\prime-\frac{1}{2}} v g_{Y M 4}^{2}}{k}=\frac{\alpha^{\prime+\frac{1}{2}} g_{Y M 4}^{2}}{k a}=\frac{\alpha^{+\frac{1}{2}} \Lambda g_{Y M 4}^{2}}{k} \\
\tilde{g}_{s}=\sqrt{\alpha^{\prime}} \Lambda g_{Y M 4}^{2}=\left(\frac{r_{0}}{\sqrt{\alpha^{\prime}}}\right) g_{Y M 4}^{2}
\end{gathered}
$$

2 The author apologizes for using the word 'dual' as a verb, but this practice has become so common that more correct substitutes such as 'perform a duality' or even 'dualize' sound awkward to contemporary ears. (Doubly so for that suspiciously, subversively foreign variant: 'dualise', c.f.,e.g. [11].) 


\section{Decoupling limit of stringy dimensional deconstruction}

This section is a straightforward inclusion of the backreaction of the branes on the geometry, and a carrying out of the usual decoupling limit which discards the asymptotic region where the backreaction can be neglected.

We point out that the geometry of the boundary is not asymptotically $A d S_{5}$ at spatial infinity. If we characterize points of spatial infinity as limits of paths with $u^{2} \equiv z^{A} z^{A}+r^{2} \rightarrow$ $\infty$ with $z^{A} / r$ and $x^{\mu}, \tilde{x}_{3}$ fixed, then the warp factor multiplying the $\left(d \tilde{x}_{3}\right)^{2}$ grows as a different power of $u$ at inifinity than does the warp factor multiplying $\eta_{\mu \nu} d x^{\mu} d x^{\nu}$.

In addition to the horizon and the boundary, the solution we discuss has a singular region at $r=z^{A}=0$ which can be interpreted neither as a threebrane horizon nor as a boundary. Locally this singularity represents an infinite, continuously distributed array of NS fivebrane charge.

One interesting fact about our background is that even though the fivebranes in the supergravity solution are smeared into a continuous distribution along the $\tilde{x}_{3}$ direction, the breaking of translational invariance to a discrete subgroup - a breaking of symmetry of which the fivebranes are ultimately the source - is still visible in the supergravity approximation. The basic physics of this has been discussed in [12]. The $H$-flux transmits the breaking of translational invariance over long distances; however fields which transmit the breaking of the discrete translational invariance preserved by the lattice are shortranged and these effects cannot be seen in the smeared supergravity solution.

To say this leaves open the question of whether the information lost by the smeared solution is necessary for computing amplitudes in this background. It is certainly true that in the undecoupled solution, giving a vev to modes living on the NS fivebranes (T-dual to the twisted sectors in the $\mathbb{Z}_{k}$ orbifold) alters the dynamics of the threebrane probe. It seems plausible that the interaction of the threebranes with some subset of these modes survives the decoupling limit. In the holographic correspondence, these modes would then be to frozen, non-normalizable modes corresponding to perturbations of the gauge theory Hamiltonian which break the discrete translational invariance of the lattice. The novelty would be that these modes would be strongly supported near $r=z^{A}=0$, rather than (or as well as) at $u \rightarrow \infty$. 


\subsection{Backreaction of D2-branes at orbifolds}

First we construct the metric produced by $n k$ D3-branes distributed in a symmetric configuration on the covering space. Using the conventions of [11], we have

$$
\begin{aligned}
d s^{2}= & Z_{p}^{-\frac{1}{2}} \eta_{\mu \nu} d x^{\mu} d x^{\nu}+Z_{p}^{\frac{1}{2}} d y^{i} d y^{i} \\
& \exp \{2 \Phi\}=g_{s}^{2} Z_{p}^{\frac{(3-p)}{2}} \\
C_{(p+1)}= & \left(Z_{p}^{-1}-1\right) g_{s}^{-1} d x^{0} \wedge \cdots \wedge d x^{p}
\end{aligned}
$$

where $\mu$ ranges from 0 to $p$, and $i$ ranges from $p$ to $9 . Z_{p}$ is a harmonic function of the coordinates $y^{i}$ which we compute in the appendices.

Now, restrict to the case where $p=2$ and distribute the branes evenly over the angular direction. For large $k$ and fixed $r_{0}$, this approximation becomes very good. The smeared solution is

$$
\begin{gathered}
d s^{2}=Z_{2}^{-\frac{1}{2}} \eta_{\mu \nu} d x^{\mu} d x^{\nu}+Z_{2}^{+\frac{1}{2}} d z^{A} d z^{A} \\
+Z_{2}^{+\frac{1}{2}}\left[d r^{2}+\frac{1}{4} r^{2} d \Omega_{2}^{2}+\frac{1}{k^{2}} r^{2}\left(d \beta+A_{\phi}^{[\beta]} d \phi\right)^{2}\right]
\end{gathered}
$$

where

$$
\begin{gathered}
A_{\phi}^{[\beta]} \equiv k(1-\cos \theta), \\
d \Omega_{2}^{2}=d \theta^{2}+\sin ^{2} \theta d \phi^{2},
\end{gathered}
$$

with $Z_{2}$ computed as a superposition of the individual harmonic factors of the twobranes. Our coordinate system is defined in Appendix A and the explicit form of $Z_{2}$ is given in Appendix B.

\subsection{T-duality and decoupling limit}

After $T$-duality our new metric is:

$$
d \tilde{s}^{2}=Z_{2}^{-\frac{1}{2}}\left[\eta_{\mu \nu} d x^{\mu} d x^{\nu}+\frac{r_{0}^{2}}{r^{2}} d \tilde{x}_{3}^{2}\right]+Z_{2}^{+\frac{1}{2}}\left[d z^{A} d z^{A}+d r^{2}+\frac{r^{2}}{4} d \Omega_{2}^{2}\right]
$$

and the transformed dilaton, RR potential, and NS-NS two-form are given by

$$
\exp \{2 \tilde{\Phi}\}=\frac{\alpha^{\prime}}{G_{\gamma \gamma}} \exp \{2 \Phi\}=\frac{\alpha^{\prime} k^{2} g_{s}^{2} Z_{2}^{+\frac{1}{2}}}{r^{2} Z_{2}^{+\frac{1}{2}}}=\frac{\alpha^{\prime} k^{2} g_{s}^{2}}{r^{2}}=\frac{\alpha^{\prime} \tilde{g}_{s}^{2}}{r^{2}}=\left(\frac{r_{0}^{2}}{r^{2}}\right) g_{Y M 4}^{4}
$$




$$
\begin{gathered}
\tilde{B}_{M N} d x^{M} \wedge d x^{N}=\alpha^{\prime} \Lambda(1-\cos \theta) d \phi \wedge d \tilde{x}_{3} \\
\tilde{C}_{M N S T} d x^{M} \wedge d x^{N} \wedge d x^{S} \wedge d x^{T}=\Lambda\left(Z_{2}^{-1}-1\right) d x^{0} \wedge d x^{1} \wedge d x^{2} \wedge d \tilde{x}_{3}
\end{gathered}
$$

As before, the large $-k$ limit, with tilde'd quantities held fixed, is smooth.

Taking the decoupling limit amounts to discarding the asymptotic region. In this limit we make the replacement

$$
\begin{gathered}
Z_{2} \rightarrow Z_{2 \text { (dec.) }} \equiv \\
\frac{16 \alpha^{\prime 2} \tilde{g}_{s} n}{g_{-}^{3 / 2} g_{+}^{2}}\left(4\left(u^{2}+r_{0}^{2}\right) E_{2}\left[-\frac{8 r_{0} f r}{g_{-}}\right]-g_{+} E_{1}\left[-\frac{8 r_{0} f r}{g_{-}}\right]\right) .
\end{gathered}
$$

$E_{1,2}$ and $g_{ \pm}$are defined in the appendices.

We have constructed our gauge theory to flow in the infrared to the maximally supersymmetric $U(n)$ gauge theory in four dimensions. Therefore it should not surprise us that the background we have constructed contains a horizon whose near-horizon geometry is precisely $A d S_{5} \times S^{5}$. To see this, simply scale towards the point $\phi=z^{A}=0, \theta=\delta, r=r_{0}$. The backreaction factor $Z_{2}$ behaves in this limit as

$$
Z_{2} \propto \Delta^{-4}
$$

where $\Delta$ is the distance to the threebranes as computed with the type IIB metric without the backreaction included.

\section{Bulk signatures of the boundary's spatial discreteness}

\subsection{Worldsheet instantons as umklapp effects}

The most noticeable feature of lattice gauge theory is, of course, its spatial discrete-

ness. There is no obvious sign of this discreteness in the gravitational background we have constructed. The reader may object that this is because we have simply used the wrong metric to describe our space: after all, the geometry of the D3-NS5 system is spatially inhomogeneous in the $\tilde{x}_{3}$ direction, something which the 'smeared' solution we wrote down does not accurately reflect. 


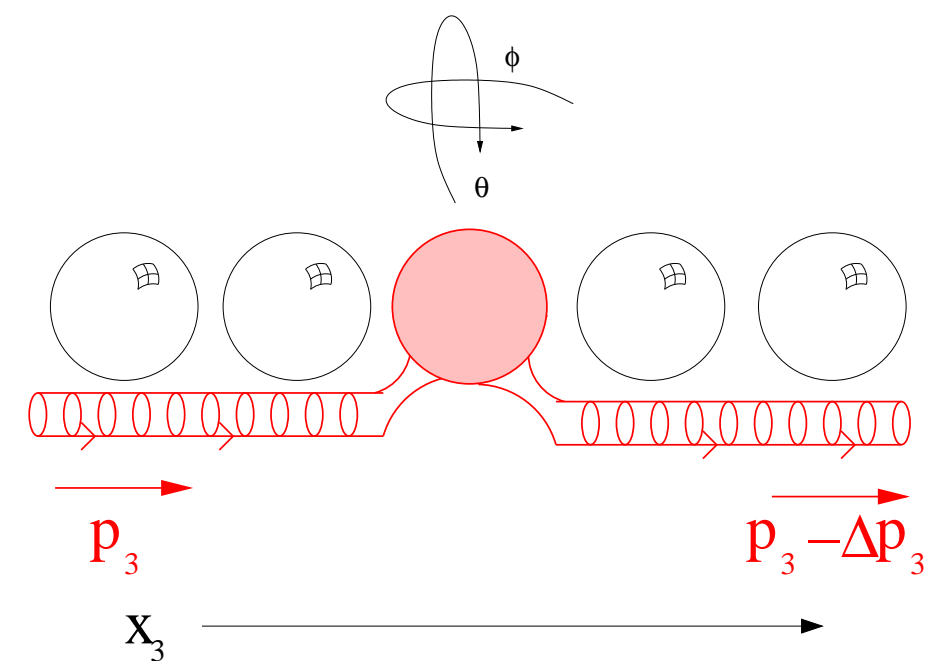

Fig. 3: A worldsheet instanton process in which a closed string wraps a twosphere and loses an amount $\Delta \tilde{p}_{3}=\Lambda$ of momentum in the $\tilde{x}_{3}$ direction. This process gives rise to gauge theory amplitudes which violate momentum conservation.

However as we shall see we do not need a solution with localized NS fivebranes to find momentum-nonconserving processes in the bulk theory. Worldsheet instanton processes encode the violation of momentum conservation in precisely the units we expect, despite the fact that the solution is translationally invariant.

Note that the conserved momentum of a string is not simply its mechanical momentum

$$
P_{3} \neq P_{3(\text { mech })}=\frac{1}{2 \pi \alpha^{\prime}} \oint d \sigma^{1} G_{\tilde{x}_{3} M} \dot{X}^{M}
$$

The string is coupled minimally to the NS-NS $B$-field, and the conserved quantity is the generalized momentum, which has a term:

$$
P_{3}=P_{3(\mathrm{mech})}+\frac{1}{2 \pi \alpha^{\prime}} \oint d \sigma^{1} B_{\tilde{x}_{3} M} \partial_{1} X^{M}
$$

There is only a sensible conserved $\tilde{x}_{3}$-momentum if the $B$-field is single-valued on the $S^{2}$ and independent of $\tilde{x}_{3}$. For the background we consider there is no gauge in which $B_{M N}$ is both. We choose a gauge in which $B_{M N}$ is independent of $\tilde{x}_{3}$ but not single valued. As a result the momentum in the $\tilde{x}_{3}$ direction will be conserved except when there are worldsheet process which sense the non-single-valuedness of the gauge field - that is, momentum conservation will be violated by string instanton processes. 
We now compute the momentum loss from a worldsheet instanton in the type IIB string background we have constructed. The coupling of the $B$-field to the worldsheet is of the form

$$
\frac{1}{4 \pi \alpha^{\prime}} \int d^{2} \sigma \epsilon^{a b} \partial_{a} X^{\mu} \partial_{b} X^{\nu}
$$

If the $B$ field is given by $B_{\tilde{x}_{3} \phi}=-B_{\phi \tilde{x}_{3}}=\alpha^{\prime} \Lambda(1-\cos \theta)$ then the $B$-field contribution to the momentum $P_{3}$ of the string is

$$
P_{3}=P_{3(\text { mech })}+\frac{1}{4 \pi \alpha^{\prime}} \oint d \sigma^{1} B_{\tilde{x}_{3} \phi} \phi^{\prime}
$$

Fortunately the backraction factor $Z_{2}$ does not enter into $B_{M N}$. The momentum loss is given by the integral

$$
\begin{gathered}
\Delta P_{\tilde{x}_{3}}=\frac{1}{4 \pi \alpha^{\prime}} \Delta \oint d \sigma^{1} B_{\tilde{x}_{3} \phi} \phi^{\prime} \\
=\frac{1}{4 \pi \alpha^{\prime}} \int d \sigma^{0} d \sigma^{1} B_{\tilde{x}_{3} \phi, M} \phi^{\prime} \dot{X}^{M}=\frac{1}{4 \pi \alpha^{\prime}} \int d \sigma^{0} d \sigma^{1} B_{\tilde{x}_{3} \phi, M}\left(\phi^{\prime} \dot{X}^{M}-\dot{\phi} X^{M^{\prime}}\right) \\
=-\frac{1}{4 \pi \alpha^{\prime}} \int d\left[B_{\tilde{x}_{3} M} d X^{M}\right]=\frac{\Lambda}{4 \pi} \int d \phi \wedge d \theta \sin \theta=\Lambda
\end{gathered}
$$

which means the amount of $\tilde{x}_{3}$ momentum lost by the string is quantized in units of the inverse lattice spacing, as one would anticipate in a theory with a lattice cutoff.

We point out that as one would expect, this process is highly suppressed near the threebrane horizon, where instanton action diverges, being proportional to the area of the $S^{2}$, which agrees with our expectation that momentum-violating processes should be suppressed at low energies.

\subsection{Point-particle analog}

In order better to understand this odd effect, consider the point-particle analog in which we have an electric dipole moving on a cylinder in a constant magnetic field $B$. Let the axial and angular coordinates of the cylinder be given by $z$ and $\phi$, respectively. We want to pick a gauge in which the gauge potential is independent of $z$. One such gauge is $A_{\phi}=0$, in which the angular momentum of the particle has its naïve definition. Then the gauge potential is $A_{z}=B \phi$. In this gauge the momentum in the $z$ direction is not single valued; it changes by $2 \pi g B$ as the particle makes one circuit around the $\phi$ direction.

We can imagine a process in which the dipole breaks apart and one of the two charged particles traverses the circle, then binds to its partner again. In such a process, the momentum of the system changes by precisely $\Delta p_{z}= \pm 2 \pi g B$. To an observer unable to 
resolve the internal structure of the dipole, such an effect would be indistinguishable from the effect of a periodic spatial inhomogeneity of periodicity $\Delta z=\frac{1}{g B}$.

How can this be? What about Nöether's theorem? Nöether's theorem, which normally guarantees momentum conservation in a translationally invariant system, just never applies to the system we consider: in a gauge in which there is a Lagrangian, the Lagrangian depends on $z$, and in any gauge in which the gauge field is $z$-independent, the Lagrangian does not exist as a single-valued function on configuration space.

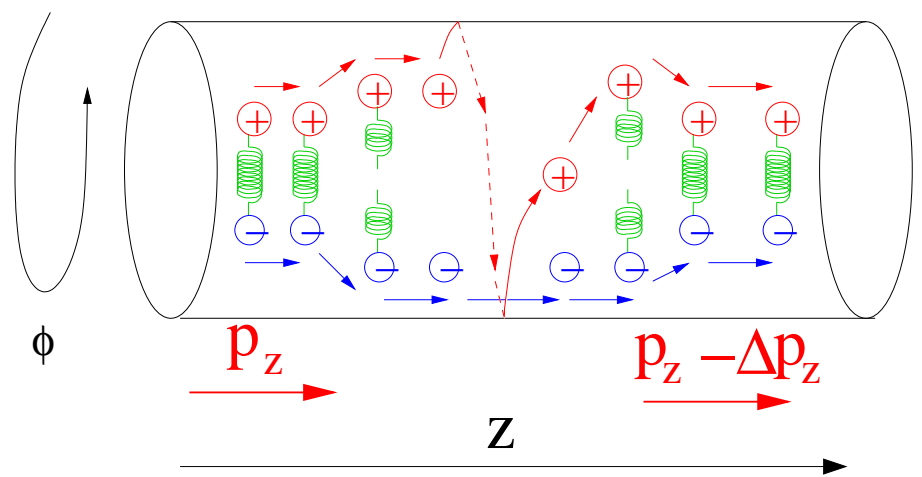

Fig. 4: An electric dipole in a magnetic field can lose momentum even if the system is spatially homogeneous.

One can learn more by trying to apply Nöether's theorem to the system of dipoles coupled to a dynamical electromagnetic field on the cylinder. In this system, there is no explicit breaking of translational invariance: there really is a conserved stress tensor, the integral of whose $T_{z}^{0}$ component would ordinarily be a conserved momentum. However momentum conservation is spontaneously broken by the presence of the background magnetic flux. The generator of translations contains a Poynting term $\Delta P_{z}=E^{\theta} \cdot B$, and since $B$ has a vev, the momentum has nonvanishing Poisson brackets with $A^{\theta}$, shifting the gauge field by an amount proportional to $B$. Thus translational invariance in this system is realized nonlinearly. (Physically, a charged particle which goes around the $\theta$ direction leaves behind one unit $g$ of electric flux, which contributes exactly $\mp g B$ to the Poynting momentum.) The same resolution applies to our type IIB string background.

\section{Giant gravitons and bounded momentum for one-particle states}

In addition to giving rise to umklapp processes whereby gauge theory composites can lose momentum, the lattice also imposes an upper bound $P_{\max }$ on composite states. Since 
composites can be made up of large numbers of partons at large 't Hooft coupling, we know that $P_{\max }$ can in principle be parametrically larger than $\Lambda$. Is there any way to derive the bound $P_{\max }$ by considering the dual gravitational theory?

Qualitatively, the situation is rather close to that of [13]. The presence of the $H$ flux makes the closed fundamental string want to blow up and wrap an $S^{1}$ of the $S^{2}$ parametrized by $\phi$ and $\theta$.

In the spirit of [13] we now estimate the maximum momentum of a closed fundamental string wrapping a circle of the $S^{2}$. The reduced symmetry of this background makes the problem harder than the analogous problem for giant gravitons with large angular momentum in $A d S_{p} \times S^{q}$. Nonetheless we find that the same basic physical process is at work in this system as in those of [13]. We will find that the maximal momentum of a single particle state is proportional to $\Lambda$ with a coefficient of order unity, with no powers

of $n$ or the 't Hooft coupling $\lambda \equiv\left(g_{Y M 4}^{2} n\right)^{\frac{1}{2}}$; that is, the perturbative bound for single free quanta will also apply, up to a non-large numerical factor, to multi-parton composites in the strongly interacting theory.

\subsection{Equations of motion}

The lagrangian on the closed string worldsheet is

$$
S=-\frac{1}{4 \pi \alpha^{\prime}} \int d^{2} \sigma\left[\sqrt{-g} g^{a b} G_{M N}(X)+\epsilon^{a b} B_{M N}(X)\right] \partial_{a} X^{M} \partial_{b} X^{N}+\alpha^{\prime} \Phi(X) \cdot \sqrt{-g} R
$$

with the $\epsilon$-tensor normalized such that $\epsilon^{01}=+1$. (The action is generally covariant because we define $d^{2} \sigma \equiv \frac{1}{2} \epsilon^{a b} d \sigma^{a} \wedge d \sigma^{b}$ so the action is invariant under coordinate transformations which do not preserve the volume.)

The equations of motion in unit gauge $g_{a b}=\eta_{a b}$ for the embedding coordinates are then

$$
0=\partial_{a} \partial^{a} X^{S}+\Gamma_{M N}^{S}\left(\partial_{a} X^{M}\right)\left(\partial^{a} X^{N}\right)-\frac{1}{2} H_{M N}{ }^{S} \epsilon^{a b}\left(\partial_{a} X^{M}\right)\left(\partial_{b} X^{N}\right)
$$

where

$$
H_{M N S} \equiv B_{M N, S}+\text { cyclic }
$$

The constraint is

$$
0=G_{M N} \partial_{a} X^{M} \partial^{a} X^{N}-\alpha^{\prime} \partial_{a} \partial_{b} \Phi
$$

We will now study an approximation to the actual giant graviton problem in which the metric on the sphere is round and its size is constant; this will capture most of the concepts involved. 


\subsection{Giant gravitons on $R^{1,1} \times S^{2}$}

We examine a toy model of our problem in which the size of the sphere is fixed, the $H$-flux and dilaton are constant, and the only spacetime coordinates are $X^{0}, X^{3}, \phi$, and $\theta$. We need not trouble ourselves that such a background does not represent a solution to the spacetime equations of motion; the only symptom is the nonvanishing worldsheet $\beta$-function, and since we will be considering only classical solutions this inconsistency will not impinge on our discussion.

We take the metric be

$$
d s^{2}=-\left(d x^{0}\right)^{2}+\left(d x^{3}\right)^{2}+R^{2}\left[d \theta^{2}+\sin ^{2} \theta d \phi^{2}\right]
$$

and so the maximum where $R$ is a fixed radius that does not depend on any of the other coordinates. We also assume that only the azimuthal angle $\phi$ depends on the worldsheet spatial coordinate $\sigma^{1}$ and the rest depend only on worldsheet time $\sigma^{0}$.

Again taking unit gauge we find

$$
\begin{gathered}
S=\frac{1}{4 \pi \alpha^{\prime}} \int d \sigma^{0} d \sigma^{1}\left[-\left(\dot{X}^{0}\right)^{2}+\left(\dot{X}^{3}\right)^{2}+R^{2} \dot{\theta}^{2}-R^{2} \sin ^{2} \theta \phi^{\prime 2}+H(1-\cos \theta) \phi^{\prime} \dot{X}^{3}\right] \\
\text { +terms which don't affect the solutions we'll be examining }
\end{gathered}
$$

The equations of motion are:

$$
\begin{array}{cr}
\dot{X}=\text { const. } & \frac{\partial}{\partial \sigma^{0}}\left(\dot{X}^{3}+H(1-\cos \theta) \phi^{\prime}\right)=0 \\
\phi^{\prime \prime}=0 & \frac{\partial^{2} \theta}{\left(\partial \sigma^{0}\right)^{2}}=\sin \theta\left(\frac{1}{2} H \dot{X}^{3} \phi^{\prime}-R^{2} \cos \theta \phi^{\prime 2}\right)
\end{array}
$$

If we further assume that $\theta$ is time-independent, then we find

$$
\begin{gathered}
X^{0}=\alpha^{\prime} E \sigma^{0}=\alpha^{\prime} E v \sigma^{0} \\
\quad E v=\alpha^{\prime} P_{3}-w H(1-\cos \theta) \\
\phi=w \sigma^{1} \\
\cos \theta=\frac{\alpha^{\prime} H P_{3}-w H^{2}}{2 R^{2} w-w H^{2}}
\end{gathered}
$$

where we have allowed for an arbitrary nonzero number $w$ of windings around the $\phi$ direction.

Since $\cos \theta$ always lies between -1 and +1 , this immediately tells us that the momentum of a giant graviton must satisfy

$$
\left|P_{3}\right| \leq \frac{2 R^{2}|w|}{\alpha^{\prime}|H|}
$$


So the number of momentum units per string is limited by the quantity $\frac{R^{2}}{H}$.

Now we will assume that there is only a single spacetime scale in the classical worldsheet problem. This will actually be true of our solution, as we shall see in the next section. (The string scale only controls the size of quantum corrections, not the classical behavior of the string.) So in our toy model we will imagine that $H$ is not too different in size from $R$. (In particular, in our model $H=\alpha^{\prime} \Lambda=r_{0}$, and we expect the dynamics of the giant graviton to prefer a point where the radius of the $S^{2}$ is also $R \sim r_{0}$, since there is no other scale for it to choose.)

So we have

$$
P_{3}-\Lambda=c \Lambda \cdot \cos \theta
$$

where $c$ is some constant of order unity. Depending on the actual value of $c$, the blown-up string state may or may not exist for all possible values of $P_{3}$. But no matter what $c$ may be, the giant solution will always exist for values of $P_{3}$ sufficiently close to $\Lambda$ ! So despite our lack of a solution to the full problem the one thing we know is that near the cutoff $\Lambda$, the fast-moving hadron always has a blown-up string solution.

The relative stability of the giant and pointlike states with equal $P_{3}$ depends on the ratio $R / H$ and so again reduces to a problem of classical dynamics, yet to be solved.

\subsection{Scalings in the actual giant graviton problem}

Though we have not been able to find the actual giant graviton solution in the actual IIB background we are considering, we can predict its coupling dependence, if it does indeed exist (which we shall assume in this section).

We consider a nontrivial warp factors to the sphere, $X^{0}$, and $X^{3}$ terms in the metric; as in the actual model we consider, these warp factors do not depend on $x^{0}, x^{3}$, or $\phi$, but they may depend on $\theta$. At this level of generality, $\theta$ is distinguished from the other coordinates only in that the $B_{M N}$ field depends only on it and not on any other coordinate. So we will denote all the other coordinates, including $\theta$, by $\left\{t^{a}\right\}=\left\{t^{0} \equiv \theta, t^{1}, t^{2}, \cdots\right\}$.

Let us now fix the dependence on the 't Hooft parameter $\lambda \equiv\left(g^{2} n\right)^{\frac{1}{2}}$. We will let the coefficient of the metric in the $x^{\mu}$ directions scale as $\lambda^{-1}$, the coefficient of the metric in the other directions scale as $\lambda^{+1}$, and the $B_{M N}$ term scale as $\lambda^{0}$, just as in our specific problem. We have

$$
d s^{2}=-\lambda^{-1} f_{1}\left(d x^{0}\right)^{2}+\lambda^{-1} f_{2}\left(d x^{3}\right)^{2}+\lambda^{+1} f_{3} d \phi^{2}+\lambda^{+1} h_{\alpha \beta} d t^{\alpha} d t^{\beta}
$$


The worldsheet action is

$$
S=\frac{1}{4 \pi \alpha^{\prime}} \int d \sigma^{0} d \sigma^{1}\left[\lambda^{-1}\left(-f_{1}\left(\dot{X}^{0}\right)^{2}+f_{2}\left(\dot{X}^{3}\right)^{2}\right)+H \dot{X}^{3} \phi^{\prime}(1-\cos \theta)+\lambda^{+1}\left(h_{\alpha \beta} \dot{t}^{\alpha} \dot{t}^{\beta}+f_{3} \phi^{\prime 2}\right)\right]
$$

Now perform the rescaling $X^{0,3} \equiv \lambda^{+1} \tilde{X}^{0,3}$. Then the worldsheet action is:

$$
S=\frac{\lambda}{4 \pi \alpha^{\prime}} \int d \sigma^{0} d \sigma^{1}\left[f_{1}\left(\dot{\tilde{X}}^{0}\right)^{2}+f_{2}\left(\dot{\tilde{X}}^{3}\right)^{2}+H \dot{\tilde{X}}^{3} \phi^{\prime}(1-\cos \theta)+h_{\alpha \beta} \dot{t}^{\alpha} \dot{t}^{\beta}+f_{3} \phi^{\prime 2}\right]
$$

The energy and momenta are defined by

$$
\begin{gathered}
\tilde{E}=-\frac{\lambda f_{1}}{\alpha^{\prime}} \dot{\tilde{X}}^{0} \quad \tilde{P}_{3}=\frac{\lambda f_{2}}{\alpha^{\prime}}\left(\dot{\tilde{X}}^{3}-H(1-\cos \theta)\right) \\
p_{\alpha}=\frac{\lambda}{\alpha^{\prime}} h_{\alpha \beta} \dot{t}^{\beta}
\end{gathered}
$$

The only place $\lambda$ appears in the action is as an overall constant mutlitplying all terms uniformly. Since the problem of the existence of a giant graviton solution is strictly a classical worldsheet problem, $\lambda$ drops out of the problem completely. Furthermore, the only spacetime scale appearing in the classical action is $H=\alpha^{\prime} \Lambda=r_{0}$. Therefore on dimensional grounds alone, we can conclude that the maximum value of $\dot{\tilde{X}}^{3}+\frac{1}{2} H \phi^{\prime}(1-$ $\cos \theta)$ is of order $r^{0}$, and therefore $\tilde{P}_{\max } \propto \frac{\lambda r_{0}}{\alpha^{\prime}}=\lambda \Lambda$, which means

$$
P_{\max } \propto \Lambda
$$

with constant of proportionality of order 1. (We have not included the varying dilaton in this discussion. Its effect is to change the energy of the giant graviton by altering the constraint equation, and in principle this could effect the stability of the solution. However the terms it contributes to $E^{2}$ scale with the same power law in $\lambda$ as do terms which are already included.)

This is somewhat unexpected. Of course the maximal momentum of a single-particle state in a weakly coupled theory should indeed scale like $\Lambda$, but it is far more surprising that a composite state in a confining theory (or even a 'marginally confining' theory with vanishing beta function in the infrared such as ours) should behave this way. Nä̈vely, a composite with a large number $b$ partons in it (with $b$ of order $n$, say) should be able to have momentum of order $b \Lambda$.

But such a state could not be entirely stable. Any state with momentum $>>\Lambda$ can decay via umklapp's. The giant graviton would represent an endpoint of many of these 
umklapp's in which a large number of soft quanta share an amount of momentum of order $\Lambda$, with total energy of order $\Lambda$.

It is possible that in our particular system the giant graviton states, like the Bloch wave excitations of the perturbative gauge theory, are actually BPS-saturated and as a result the momentum cutoff and dispersion relation are exactly the same as for single quanta of the fundamental fields of the system, namely

$$
E_{B P S}=\frac{\Lambda}{\pi} \sin \frac{\left|\pi P_{3}\right|}{\Lambda}
$$

If so, this is quite interesting, as the bound is a non-additive BPS bound for strongly interacting composite particles with momentum along a direction with no continuous translational invariance! (Other recent insights gained into lattice supersymmetry from the ideas of dimensional deconstruction include [14], [15]).

It would be interesting to find a fill out a field-theoretic picture of a giant graviton state, perhaps by computing how the momentum is shared among its many constituents.

\section{Conclusions}

We have constructed a gravitational dual of a theory with $2+1$ continuous and one discrete dimension, all of infinite extent. We have resolved apparent paradoxes that stem from an exact equivalence between a gauge theory in a discrete spacetime and a gravitational theory on a continuous background. As in other manifestations of gravity/gauge theory duality, one can use supergravity to learn about the large- $n$ limits of gauge theory, or on the other hand use the a priori well-definedness of gauge theory to extend the domain of definition of quantum gravity. The construction of supergravity duals for discretized field theories promises interesting progress along both lines.

\subsection{The relevance of discretization}

Lattice gauge theory is a tool which is difficult to use in practice. The lattice makes it possible to ask, and sometimes answer, questions about the behavior of gauge theories without appealing to a perturbative expansion. Relating the behavior of the lattice theory to that of the continuum theory is difficult because the discretization at the scale $\Lambda$ contributes corrections to the effecive action of the continuum theory which break Lorentz invariance and other symmetries which one wishes to restore in the infrared. 
The usefulness of the lattice theory for describing Yang-Mills theory then rests on the irrelevance of symmetry-breaking operators. It is not known how to compute the dimensions of these operators in the infrared with conventional methods, since the gauge theory is strongly coupled there.

The gravitational description, however, makes it possible to read off the dimensions of these operators at large $n$ by computing the eigenvalues of the Laplacian acting on linearized fluctuations of bulk fields about the solution we have described. This program could be carried out not only for the $N=4, D=4$ theory but for many other strongly coupled gauge theories if the supergravity duals of their discretized versions can be found.

\subsection{Brane freeze: fixing the gauge coupling and lattice spacing}

One point to keep in mind is the relationship between the lattice theory described in this paper and the type of system conventionally referred to as 'lattice gauge theory'. At first sight the two appear quite different, even after allowing for the presence of the massless adjoint matter and supersymmetry. Our system has the additional oddity that the $U(1)$ degree of freedom, which in the continuum theory is completely decoupled, here couples to the branes through a nonrenormalizable contribution to the kinetic term for the gauge fields:

$$
L_{Y M 4}=\frac{1}{4 g_{Y M 4}^{2}} \operatorname{tr}\left[\left(1-\frac{\hat{Y}^{r}}{2 \Lambda}\right)\left(\hat{F_{\mu \nu}} \hat{F}^{\mu \nu}\right)\right]+\cdots
$$

where $\hat{Y}^{r}$ is a linear combination of the hypermultiplet scalars in the gauge theory which corresponds to infinitesimal inward motion in the $r$ direction.

We chose our decoupling limit in such a way that the gauge coupling in the far infrared; at first sight, then, it appears strange that the gauge coupling can be changed to any arbitrary value by a shift, but it merely reflects the fact that the dilaton runs as a function of the distance from the origin $r=0$.

The same comments that apply to the gauge coupling also apply to the lattice spacing. If we shift $\hat{Y}^{r}$ we change the effective lattice spacing as well. This may appear somewhat paradoxical, since we have shown in a previous section that the violation of conservation of $\tilde{x}_{3}$-momentum is strictly quantized in units of $\Lambda$. The resolution is that there is that the action $L_{Y M 4}$ contains Lorentz-noninvariant terms such as

$$
\left[\operatorname{tr}\left(1-\frac{\hat{Y}^{r}}{2 \Lambda}\right)\left(\partial_{\tilde{x}_{3}} \hat{X}^{i}\right)\left(\partial_{\tilde{x}_{3}} \hat{X}^{i}\right)\right]
$$


which change the kinetic terms for all the fields in the 3 direction when we shift $\hat{Y}^{r}$. The theory is still Lorentz-invariant in the infrared, but with a different assignment of Lorentz transformations. After we shift $\hat{Y}^{r}$, a rescaled $\tilde{x}_{3}^{[\text {new] }}$ coordinate which enters into a four-vector with $x^{0,1,2}$. The violation of $P_{\tilde{x}_{3}^{[n e w]}}$ and the violation of $P_{\tilde{x}_{3}}$ are quantized in different units, which differ by the obvious rescaling.

In a conventional lattice gauge theory, we usually think of neither the tree-level gauge coupling nor the lattice spacing changes as we vary the vev of the matter fields. But when we say the words 'lattice gauge theory', we really mean any one of an infinite family of theories which differ by nonrenormalizable couplings such as the very special ones that provide the effects described above. The theory we have been discussing is indeed in the universality class of a conventional gauge theory, but the presence of the massless scalars simply highlights the fact that irrelevant terms can endow an effective theory with dramatic effects.

Nonetheless, in order to come closer to conventional lattice gauge theory, one may want to provide masses to some (or all) of the scalars of the system, perhaps preserving $N=1$ or $N=2$ supersymmetry in the process. One inviting possibility for future research is to perturb our background with fluxes along the lines of [16] in a way which freezes the branes in their equilibrium positions at $r=r_{0}$. More precisely, the perturbation on the IIA side would look like a $k \underline{\text { th }}$ order polynomial perturbation of the dilaton and $p$-form fields on the covering space of the $\mathbb{Z}_{k}$ orbifold with zeroes arranged in a ring around $r=0$. This solution may be obtainable via $T$-duality to the type IIB backgrounds with holomorphic axiodilaton described in [17], [18]. The combination of the superpotential deformation with the power-law running of the gauge coupling may provide interesting new phenomena for the gravity/gauge theory duality to illuminate, as well as mimicking 'lattice gauge theory' (as conventionally imagined) more closely than does the theory we have explored in the present paper.

\subsection{A general theory of holography?}

In the background we study we have taken the limit in which the number of lattice sites goes to infinity, with the lattice spacing taken to be finite. We could have discretized all spatial directions and taken the decoupling limit with the number of lattice sites held fixed, still retaining a continuum gravitational description of the system at large $n$. It would be extremely interesting to study the graviational background obtained this way, as it corresponds to a system with a large but finite number of degrees of freedom per unit 
volume. (Actually one would also have to go one step further and impose some sort of cutoff on field space at the same time). If one limited such a system to a finite number of lattice sites, the gravitational dual would have a boundary with finite area and nonzero spatial dimension. Such boundaries would look locally like Schwarzschild or deSitter horizons and may be interesting for the study of black holes, and also for the understanding of inflationary cosmology from a holographic point of view.

\section{Appendix A. Conventions about coordinates and indices}

Define $x^{\mu}, \mu=0,1,2$ to be the longitudinal coordinates along the type IIA D2-brane, $z^{A}, A=7,8,9$ to be three of the transverse coordinates, and $y^{i}, i=3,4,5,6$ to be the four other transverse coordinates, on which the orbifolding acts. The radial coordinate $r$ on $\mathbb{C}^{2}$ is defined by $r^{2} \equiv y^{i} y^{i}$ and we will sometimes use the coordinate $u$ to denote the radial coordinate on $R^{7}$, i.e. $u^{2} \equiv r^{2}+z^{A} z^{Z}$. We will use capital $X^{M}$ to denote more general coordinate systems.

Next we define four-dimensional polar coordinates on the space $R^{4}=\mathbb{C}^{2}$ spanned by the $y^{i}$ :

$$
\begin{array}{rlrl}
\phi=\tan ^{-1}\left(\frac{y_{3} y_{6}+y_{4} y_{5}}{y_{3} y_{5}-y_{4} y_{6}}\right) & y_{3} & =r \cos \left(\frac{\phi}{2}+\beta\right) \cos (\theta / 2) \\
\theta=\sin ^{-1}\left(\frac{2 \sqrt{y_{3}^{2}+y_{4}^{2}} \sqrt{y_{5}^{2}+y_{6}^{2}}}{y_{3}^{2}+y_{4}^{2}+y_{5}^{2}+y_{6}^{2}}\right) & y_{4}=r \sin \left(\frac{\phi}{2}+\beta\right) \cos (\theta / 2) \\
\beta=\frac{1}{2}\left[\tan ^{-1}\left(y_{4} / y_{3}\right)-\tan ^{-1}\left(y_{6} / y_{5}\right)\right] & y_{5}=r \cos \left(\frac{\phi}{2}-\beta\right) \sin (\theta / 2) \\
r=\sqrt{y_{3}^{2}+y_{4}^{2}+y_{5}^{2}+y_{6}^{2}} & y_{6}=r \sin \left(\frac{\phi}{2}-\beta\right) \sin (\theta / 2)
\end{array}
$$

An element $\hat{M}$ of the subgroup $S U(2)_{+}$of the rotation group $S O(4)=S U(2)_{+} \times$ $S U(2)$ - acts on these coordinates by

$$
\left[\begin{array}{l}
y_{3}+i y_{4} \\
y_{5}+i y_{6}
\end{array}\right] \rightarrow \hat{M} \cdot\left[\begin{array}{l}
y_{3}+i y_{4} \\
y_{5}+i y_{6}
\end{array}\right]
$$

In these coordinates the metric $G_{y^{i} y^{j}}=\delta^{i j}$ on $R^{4}=\mathbb{C}^{2}$ is given by

$$
d s^{2}=\eta_{\mu \nu} d x^{\mu} d x^{\nu}+d z^{A} d z^{A}+d r^{2}+r^{2}\left(d \beta+A_{\phi}^{[\beta]} d \phi\right)^{2}+\frac{1}{4} r^{2} d \Omega_{2}^{2}
$$


where

$$
\begin{gathered}
A_{\phi}^{[\beta]} \equiv 1-\cos \theta \\
d \Omega_{2}^{2}=d \theta^{2}+\sin ^{2} \theta d \phi^{2} .
\end{gathered}
$$

Now, let us instead consider a metric corresponding to an a $\mathbb{Z}_{k}$ orbifold, taking the same coordinate system. The effect of the orbifolding is to reduce the proper length of the $\beta$ direction by a factor of $k$, leaving all other metric components unchanged. The metric on the orbifold is

$$
d s^{2}=\eta_{\mu \nu} d x^{\mu} d x^{\nu}+d z^{A} d z^{A}+d r^{2}+\frac{r^{2}}{k^{2}}\left(d \beta+A_{\phi}^{[\beta]} d \phi\right)^{2}+\frac{1}{4} r^{2} d \Omega_{2}^{2},
$$

where now

$$
A_{\phi}^{[\beta]} \equiv k(1-\cos \theta)
$$

\section{Appendix B. Metric of the twobrane solution and its $T$-dual}

We obtain the metric for twobranes probing the orbifold by first taking the metric of $n k$ twobranes on the covering space, and then taking the quotient by reducing the proper size of the angular direction $\beta$ by a factor of $k$.

To obtain the metric on the covering space, we insert the backreaction factors $Z_{2}^{-\frac{1}{2}}$ and $Z_{2}^{+\frac{1}{2}}$ into the transverse and longitudinal parts of the metric [19], [11]:

$$
\begin{gathered}
d s^{2}=Z_{2}^{-\frac{1}{2}} \eta_{\mu \nu} d x^{\mu} d x^{\nu}+Z_{2}^{+\frac{1}{2}} d z^{A} d z^{A} \\
+Z_{2}^{+\frac{1}{2}}\left[d r^{2}+r^{2}\left(d \beta+A_{\phi}^{[\beta]} d \phi\right)^{2}+\frac{r^{2}}{4} d \Omega_{2}^{2}\right]
\end{gathered}
$$

where

$$
\begin{gathered}
A_{\phi}^{[\beta]} \equiv 1-\cos \theta \\
Z_{2} \equiv 1+\sum_{j=0}^{k-1} \frac{K_{2}}{\left[z^{A} z^{A}+\left(y^{i}-Y_{(j)}^{i}\right)^{2}\right]^{5 / 2}}
\end{gathered}
$$

and where we have defined

$$
K_{2} \equiv 6 \pi^{2} g_{s} \alpha^{(5 / 2)} n
$$

and

$$
\left[\begin{array}{c}
Y_{(j)}^{3}+i Y_{(j)}^{4} \\
Y_{(j)}^{5}+i Y_{(j)}^{6}
\end{array}\right] \equiv\left[\begin{array}{cc}
\exp \left\{\frac{2 \pi i j}{k}\right\} & 0 \\
0 & \exp \left\{\frac{-2 \pi i j}{k}\right\}
\end{array}\right]\left[\begin{array}{c}
Y_{(0)}^{3}+i Y_{(0)}^{4} \\
Y_{(0)}^{5}+i Y_{(0)}^{6}
\end{array}\right]
$$


are the locations of the $k$ stacks of image branes. The dilaton is

$$
\exp \{2 \Phi\}=g_{s}^{2} Z_{2}^{+\frac{1}{2}}
$$

and the $C$-field is

$$
C_{(3)}=\left(Z_{2}^{-1}-1\right) d x^{0} \wedge d x^{1} \wedge d x^{2}
$$

We perform an $S O(2)$ rotation to set $Y_{(0)}^{6}=r_{0} \cos \delta, Y_{(0)}^{8}=r_{0} \sin \delta, Y_{(0)}^{7}=Y_{(0)}^{9}=0$. We have

$$
\begin{gathered}
K_{2}^{-1}\left(Z_{2}-1\right)=\sum_{j=0}^{k-1}\left[z^{A} z^{A}+r^{2}+r_{0}^{2}\right. \\
\left.-2 r_{0} r\left\{\cos \frac{\theta}{2} \cos \frac{\delta}{2} \cos \left(\frac{\phi}{2}+\beta-\frac{2 \pi j}{k}\right)+\sin \frac{\theta}{2} \sin \frac{\delta}{2} \cos \left(\frac{\phi}{2}-\beta+\frac{2 \pi j}{k}\right)\right\}\right]^{-5 / 2} \\
=\sum_{j=0}^{k-1}\left[z^{A} z^{A}+r^{2}+r_{0}^{2}\right. \\
\left.-2 r_{0} r\left\{\cos \frac{\phi}{2} \cos \left(\frac{\theta-\delta}{2}\right) \cos \left(\frac{2 \pi j}{k}-\beta\right)+\sin \frac{\phi}{2} \cos \left(\frac{\theta+\delta}{2}\right) \sin \left(\frac{2 \pi j}{k}-\beta\right)\right\}\right]^{-5 / 2}
\end{gathered}
$$

Because we will be considering the large- $k$ limit at fixed radius $r$, the twobranes will become very closely spaced, so we smear out the $D 2$-brane charge along the $\gamma$ coordinate. So the branes are now marked by continuous values $B_{j} \sim \frac{2 \pi j}{k}$ of the $\beta$-coordinate.

So then

$$
\begin{gathered}
K_{2}^{-1}\left(Z_{2}-1\right)=\sum_{j=0}^{k-1}\left[z^{A} z^{A}+r^{2}+r_{0}^{2}\right. \\
\left.-2 r_{0} r\left\{\cos \phi \cos \left(\frac{\theta-\delta}{2}\right) \cos \left(B_{j}-\beta\right)+\sin \phi \cos \left(\frac{\theta+\delta}{2}\right) \sin \left(B_{j}-\beta\right)\right\} \quad\right]^{-5 / 2}
\end{gathered}
$$

In the sum, $\Delta j=1$ and $\Delta B=\frac{2 \pi}{k}$ so

$$
\begin{gathered}
\Delta B=\frac{\Delta B}{\Delta j}=\frac{2 \pi}{k} \\
\sum_{j} \rightarrow \int d j=\int \frac{k d B}{2 \pi}
\end{gathered}
$$

Putting in limits of integration,

$$
\sum_{j=0}^{k-1} \rightarrow \frac{k}{2 \pi} \int_{B=0}^{B=2 \pi} d B
$$


Restoring the summand/integrand we have

$$
\begin{gathered}
\sum_{j=0}^{k-1}\left[z^{A} z^{A}+r^{2}+r_{0}^{2}\right. \\
\left.-2 r_{0} r\left\{\cos \frac{\phi}{2} \cos \left(\frac{\theta-\delta}{2}\right) \cos \left(B_{j}-\beta\right)+\sin \frac{\phi}{2} \cos \left(\frac{\theta+\delta}{2}\right) \sin \left(B_{j}-\beta\right)\right\}\right]^{-5 / 2} \rightarrow \\
\left.-2 r_{0} r\left\{\cos \frac{\phi}{2} \cos \left(\frac{\theta-\delta}{2 \pi}\right) \cos (B-\beta)+\sin \frac{\phi}{2} \cos \left(\frac{\theta+\delta}{2}\right) \sin (B-\beta)\right\}\right]^{-5 / 2} \\
\left.-2 r_{0} r\left\{\cos \frac{\phi}{2} \cos \left(\frac{\theta-\delta}{2}\right) \cos B+\sin \frac{\phi}{2} \cos \left(\frac{\theta+\delta}{2}\right) \sin B\right\}\right]^{-5 / 2}+r^{2}+r_{0}^{2}
\end{gathered}
$$

Another shift in the variable $B$ of integration, this time by

$$
B \rightarrow B-\tan ^{-1}\left[\tan (\phi / 2)\left(\frac{\cos \left(\frac{\theta+\delta}{2}\right)}{\cos \left(\frac{\theta-\delta}{2}\right)}\right)\right],
$$

turns the integral into

$$
\begin{aligned}
& \frac{k}{2 \pi} \int_{0}^{2 \pi} d B\left[z^{A} z^{A}+r^{2}+r_{0}^{2}-2 r_{0} r f(\phi, \theta, \delta) \cos B\right]^{-5 / 2} \\
= & \frac{2 k}{3 \pi g_{-}^{3 / 2} g_{+}^{2}} \cdot\left(4\left(u^{2}+r_{0}^{2}\right) E_{2}\left[-\frac{8 r_{0} f r}{g_{-}}\right]-g_{+} E_{1}\left[-\frac{8 r_{0} f r}{g_{-}}\right]\right)
\end{aligned}
$$

where

$$
\begin{gathered}
f(\phi, \theta, \delta) \equiv \sqrt{\cos ^{2} \phi \cos ^{2}\left(\frac{\theta-\delta}{2}\right)+\sin ^{2} \phi \cos ^{2}\left(\frac{\theta+\delta}{2}\right)}, \\
g_{ \pm} \equiv r^{2}+z^{A} z^{A}+r_{0}^{2} \pm 4 f r_{0} r=u^{2}+r_{0}^{2} \pm 4 f r_{0} r
\end{gathered}
$$

and

$$
\begin{gathered}
E_{1}(m) \equiv \int_{0}^{\frac{\pi}{2}} \frac{d \alpha}{\sqrt{1-m \sin ^{2} \alpha}} \\
E_{2}(m) \equiv \int_{0}^{\frac{\pi}{2}} d \alpha \sqrt{1-m \sin ^{2} \alpha}
\end{gathered}
$$

are complete elliptic integrals of the first and second kind. 
This is what the branes look like on the covering space of the orbifold. On the orbifold itself, the proper length of the $\beta$-circle is reduced by a factor of $k$ relative to its length on the covering space. That is to say, keeping fixed the coordinate periodicity of $\beta$ at $\beta \sim \beta+2 \pi$, the metric is:

$$
\begin{gathered}
d s^{2}=Z_{2}^{-\frac{1}{2}} \eta_{\mu \nu} d x^{\mu} d x^{\nu}+Z_{2}^{+\frac{1}{2}} d z^{A} d z^{A} \\
+Z_{2}^{+\frac{1}{2}}\left[d r^{2}+\frac{1}{4} r^{2} d \Omega_{2}^{2}+\frac{1}{k^{2}} r^{2}\left(d \gamma+A_{\phi}^{[\beta]} d \phi\right)^{2}\right]
\end{gathered}
$$

where

$$
\begin{gathered}
A_{\phi}^{[\beta]} \equiv k(1-\cos \theta), \\
d \Omega_{2}^{2}=d \theta^{2}+\sin ^{2} \theta d \phi^{2},
\end{gathered}
$$

and

$$
Z_{2}=1+\frac{16 \alpha^{\prime 2} \tilde{g}_{s} n}{g_{-}^{3 / 2} g_{+}^{2}}\left(4\left(u^{2}+r_{0}^{2}\right) E_{2}\left[-\frac{8 r_{0} f r}{g_{-}}\right]-g_{+} E_{1}\left[-\frac{8 r_{0} f r}{g_{-}}\right]\right)
$$

The effect of the decoupling limit is to drop the constant term 1 in the backreaction factor, replacing $Z_{2}$ with

$$
\begin{gathered}
Z_{2 \text { (dec.) }}=\left(Z_{2}-1\right)= \\
\frac{16 \alpha^{\prime 2} \tilde{g}_{s} n}{g_{-}^{3 / 2} g_{+}^{2}}\left(4\left(u^{2}+r_{0}^{2}\right) E_{2}\left[-\frac{8 r_{0} f r}{g_{-}}\right]-g_{+} E_{1}\left[-\frac{8 r_{0} f r}{g_{-}}\right]\right)
\end{gathered}
$$

\section{Acknowledgements}

The author would like to thank Allan Adams, Keshav Dasgupta, John M틀eevy, Joseph Polchinski, Mohammed Sheikh-Jabbari, Stephen Shenker, Leonard Susskind, and Scott Thomas for valuable discussions. I am particularly grateful to Prof. Susskind for an explanation of the behavior of strings in discrete target spaces and for drawing my attention to existing results on the subject. I also thank the Harvard theory group for hospitality while this work was in progress. This work was supported by the DOE under contract DE-AC03-76SF00515. 


\section{References}

[1] A. Hashimoto and N. Itzhaki, "Non-commutative Yang-Mills and the AdS/CFT correspondence," Phys. Lett. B 465, 142 (1999) hep-th/9907166.

[2] J. M. Maldacena, "The large $N$ limit of superconformal field theories and supergravity," Adv. Theor. Math. Phys. 2, 231 (1998) [Int. J. Theor. Phys. 38, 1113 (1999)] hep-th/9711200.

[3] J. M. Maldacena and J. G. Russo, "Large-N Limit Of Non-Commutative Gauge Theories," Class. Quant. Grav. 17, 1189 (2000).

[4] O. Aharony, M. Berkooz, D. Kutasov and N. Seiberg, "Linear dilatons, NS5-branes and holography," JHEP 9810, 004 (1998) hep-th/9808149.

[5] L. Susskind and E. Witten, "The holographic bound in anti-de Sitter space," hepth/9805114.

[6] I. R. Klebanov and L. Susskind, "Continuum Strings From Discrete Field Theories," Nucl. Phys. B 309, 175 (1988).

[7] N. Arkani-Hamed, A.G. Cohen and H. Georgi, Phys. Rev. Lett. 86, 4757 (2001) hepth/0104005.

[8] N. Arkani-Hamed, A. G. Cohen, D. B. Kaplan, A. Karch and L. Motl, hepth/0110146.

[9] M. R. Douglas and G. W. Moore, "D-branes, Quivers, and ALE Instantons," hepth/9603167.

[10] M. R. Douglas, B. R. Greene and D. R. Morrison, "Orbifold resolution by D-branes," Nucl. Phys. B 506, 84 (1997) hep-th/9704151.

[11] C. V. Johnson, "D-brane primer," hep-th/0007170.

[12] R. Gregory, J. A. Harvey and G. W. Moore, "Unwinding strings and T-duality of Kaluza-Klein and H-monopoles," Adv. Theor. Math. Phys. 1, 283 (1997) hepth/9708086.

[13] J. M뜨reevy, L. Susskind and N. Toumbas, "Invasion of the giant gravitons from anti-de Sitter space," JHEP 0006, 008 (2000) hep-th/0003075.

[14] D. B. Kaplan, E. Katz and M. Unsal, "Supersymmetry on a spatial lattice," heplat/0206019.

[15] N. Arkani-Hamed, talk given at Strings 2002.

[16] J. Polchinski and M. J. Strassler, "The string dual of a confining four-dimensional gauge theory," hep-th/0003136.

[17] M. Grana and J. Polchinski, "Supersymmetric three-form flux perturbations on AdS(5)," Phys. Rev. D 63, 026001 (2001) hep-th/0009211

[18] M. Grana and J. Polchinski, "Gauge / gravity duals with holomorphic dilaton," Phys. Rev. D 65, 126005 (2002) hep-th/0106014

[19] G. T. Horowitz and A. Strominger, "Black Strings And P-Branes," Nucl. Phys. B 360, 197 (1991) 